Publ. Mat. 63 (2019), 715-725

DOI: 10.5565 /PUBLMAT6321909

\title{
AN INTERPOLATION PROPERTY OF LOCALLY STEIN SETS
}

\author{
VIOREL VÂJÂITU
}

\begin{abstract}
We prove that, if $D$ is a normal open subset of a Stein space $X$ of pure dimension such that $D$ is locally Stein at every point of $\partial D \backslash X_{\mathrm{sg}}$, then, for every holomorphic vector bundle $E$ over $D$ and every discrete subset $\Lambda$ of $D \backslash X_{\mathrm{sg}}$ whose set of accumulation points lies in $\partial D \backslash X_{\mathrm{sg}}$, there is a holomorphic section of $E$ over $D$ with prescribed values on $\Lambda$. We apply this to the local Steinness problem and domains of holomorphy.
\end{abstract}

2010 Mathematics Subject Classification: 32F10.

Key words: Stein space, domain of holomorphy, $\bar{\partial}$-problem.

\section{Introduction}

Stein spaces are the fundament of the theory of Functions of Several Complex Variables. Their cohomology groups with coefficients in coherent analytic sheaves are trivial in positive dimension (Cartan's Theorem B) so that the standard machinery of exact sequences implies that, for every discrete sequence $\left\{x_{\nu}\right\}$ of $X$ there is a holomorphic function $f$ on $X$ with prescribed values on $\left\{x_{\nu}\right\}$, that is Stein spaces enjoy a nice interpolation property.

In this circle of ideas an important question is the characterization of locally Stein open subsets of Stein spaces. A definitive answer is not known, so one would like to know "how far are they from being Stein".

Our main result is the following theorem. (For a complex space $X$, $X_{\mathrm{sg}}$ denotes the singular locus of $X$.)

Theorem 1. Let $X$ be a Stein space of pure dimension. Let $D \subset X$ be an open set and $\pi: E \longrightarrow D$ a holomorphic vector bundle. Suppose that $D$ is normal and locally Stein at every point of $\partial D \backslash X_{\mathrm{sg}}$.

Then, for every sequence $\left\{x_{\nu}\right\}$ of $D \backslash X_{\mathrm{sg}}$ whose set of accumulation points lies in $\partial D \backslash X_{\mathrm{sg}}$, and for every set of vectors $\xi_{\nu} \in E_{x_{\nu}}=\pi^{-1}\left(x_{\nu}\right)$, there is a holomorphic section $\sigma$ of $E$ over $D$ such that $\sigma\left(x_{\nu}\right)=\xi_{\nu}$ for all $\nu$. 
Remark. Theorem 1 remains true if $X$ is normal and " $D$ is a domain of holomorphy in $X$ " is substituted for " $D$ is locally Stein at points of $\partial D \backslash X_{\mathrm{sg}}$ "; see the subsequent Corollary 3. (For definitions see the end of this section.)

A crucial ingredient to settle Theorem 1 is the following result whose proof is given in $\S 3$.

Proposition 1. Let $X$ be a Stein space of pure dimension and let $D$ be an open subset of $X$ that is locally Stein at every boundary point of $\partial D \backslash X_{\mathrm{sg}}$. Then $D \backslash X_{\mathrm{sg}}$ admits a complete Kähler metric.

Corollary 1. Let $X$ be a Stein space of pure dimension and $D \subset X$ an open set that is normal and locally Stein at every point of $\partial D \backslash X_{\mathrm{sg}}$. Then, for every sequence $\left\{x_{\nu}\right\}$ of points in $D \backslash X_{\mathrm{sg}}$ converging to a point of $\partial D \backslash X_{\mathrm{sg}}$ and every sequence $\left\{c_{\nu}\right\}$ of complex numbers, there is a holomorphic function $h$ on $D$ with $h\left(x_{\nu}\right)=c_{\nu}$ for all $\nu$.

This corollary improves Theorem 3.1 in [12] where $h$ is asked only to be unbounded on the given sequence. Besides it allows us to remove the relative compactness hypothesis on $D$ in several known results ([2, Theorems 3.3, 3.4, and 3.14]; [15, Propositions 5.5 and 5.8, and Corollary $5.16]$ ), so for the benefit of the reader we restate them subsequently.

When $D$ is relatively compact, Corollary 1 is stated without proof in [6, Theorem 3.2.1] and it is proved in [15, Proposition 5.3].

Corollary 2. Let $X$ be a normal Stein space of pure dimension $n$ and $D \subset X$ an open set such that $\mathcal{O}(D)$ is a Stein algebra. Then $D$ is Stein in each of the following cases:

a) $D$ is locally Stein or an increasing union of Stein open subsets.

b) $D$ is a domain of holomorphy and $n=2$.

c) $D$ satisfies the strong hypersection condition and $n \geq 3$.

Note that part b) is false if $n \geq 3$. To give an example, we let $C$ be a compact Riemann surface and consider $\pi: L \longrightarrow C$ be a holomorphic vector bundle of rank $n-1$ that is negative in the sense of Grauert. Let $\tau: L \longrightarrow X$ be the blowing down of its zero section $Z \simeq C$. Then $X$ is a normal Stein space of dimension $n$ with only a singular point $\left\{x_{0}\right\}=$ $\tau(C)$. Take a point $\alpha \in C$ and put $H=\tau\left(\pi^{-1}(\alpha)\right)$. Clearly $H$ is a complex hypersurface of $X$ and $D:=X \backslash H$ is biholomorphic to $\pi^{-1}(C \backslash$ $\{\alpha\}) \backslash Z$, which is not Stein since $Z \backslash \pi^{-1}(\alpha)$ has codimension $n-1 \geq 2$ in the Stein manifold $M:=\pi^{-1}(C \backslash\{\alpha\})$. Observe also that $\mathcal{O}(D)$ is a Stein algebra isomorphic to $\mathcal{O}(M)$, and $D$ is a domain of holomorphy in $X([\mathbf{9}]$ or Corollary 3 below $)$. 
Corollary 3. In a normal Stein space $X$ an open subset $D$ is a domain of holomorphy if, and only if, $D$ is locally Stein at every point of $\partial D \backslash X_{\mathrm{sg}}$ and $\partial D \backslash X_{\mathrm{sg}}$ is dense in $\partial D$.

Corollary 4. Every increasing union of Stein open subsets of a normal Stein space is a domain of holomorphy.

Corollary 5. A normal Stein space $X$ such that $\mathcal{O}(X)$ is a Stein algebra is Stein provided that it is a locally trivial holomorphic bundle with Stein base and fiber a bounded Stein open set of some complex euclidean space.

We conclude this section with a few definitions. An open subset $D$ of a complex space $X$ is said to be locally Stein at a point $x_{0} \in \partial D$ if there is a Stein open neighborhood $U$ of $x_{0}$ such that $U \cap D$ is Stein. Also it is said that $D$ satisfies the hypersection condition if, for every Stein open subset $U$ of $X$ and every complex hypersurface $H$ of $U, H \cap D$ is Stein $[\mathbf{2}]$.

The notion of domain of holomorphy for domains in complex manifolds adapts to normal complex spaces $[\mathbf{7}],[\mathbf{1 0}]$. For not normal complex spaces it is discussed at large in [15].

A topological $\mathbb{C}$-algebra $\mathcal{A}$ is called a "Stein algebra" if $\mathcal{A}$ is topologically isomorphic to $\mathcal{O}(Z)$ for some Stein space $Z$.

\section{Linear spaces}

By definition (see [5, p. 50]) a complex space $L$ (not necessarily reduced) is said to be a linear space over another complex space $X$ if $L$ is a unitary $X \times \mathbb{C}$-module in the category of complex spaces over $X$, in other words is a complex space $L \longrightarrow X$ over $X$ together with compositions

a) $+: L \times_{X} L \longrightarrow X$ and

b) $\cdot(X \times \mathbb{C}) \times_{X} L=\mathbb{C} \times L \longrightarrow L$,

such that the module axioms hold. Roughly speaking, a linear space can be thought of as a "vector bundle with singularities".

Alternatively (see [8]), we have the following local description, namely there is an open covering of $X$ by sets $U$ such that, over each $U$ we have a presentation:

$$
\left.L\right|_{U} \longrightarrow U \times \mathbb{C}^{m} \longrightarrow U \times \mathbb{C}^{n}
$$

where the holomorphic map from $U \times \mathbb{C}^{m}$ into $U \times \mathbb{C}^{n}$ which sends $(z, \xi)$ into $(z, F(z) \cdot \xi)$ is induced by a $n \times m$ matrix whose entries $f_{j k}$ are holomorphic functions on $U$ and $\left.L\right|_{U}$ is the complex subspace of $U \times \mathbb{C}^{m}$ defined by the ideal generated by the holomorphic functions

$$
z_{1} f_{j 1}+\cdots+z_{m} f_{j m}, \quad j=1, \ldots, n .
$$


To every linear space $L \longrightarrow X$ we associate in a functorial way its coherent analytic sheaf of germs of holomorphic sections in $L$. This correspondence establishes a contravariant functor from the category of linear spaces over $X$ to the category of coherent analytic sheaves on $X$ that is an antiequivalence of categories.

The subset $W_{L}$ of $X$ of those points admitting an open neighborhood $U$ such that $\left.L\right|_{U}$ is a vector bundle, is a Zarisky dense open subset of $X$; hence $X \backslash W_{L}$ is a rare analytic subset of $X$.

Now suppose that, for every point $x$ of $X$ we have a hermitian metric $h_{x}$ on $L_{x}$. Then $h=\left\{h_{x}\right\}_{x \in X}$ is a hermitian metric on $L$ according to Grauert and Riemenschneider [8], if there is an open neighborhood $U$ of each point $x_{0}$ of $X$, an embedding $\left.L\right|_{U} \subset U \times \mathbb{C}^{q}$ and a positively definite hermitian form

$$
\widehat{h}=\sum \widehat{h}_{j k} w_{j} \bar{w}_{k}
$$

on $U \times \mathbb{C}^{q}$ with $C^{\infty}$ functions $\widehat{h}_{j k}$ on $U$ such that $h_{x}=\left.\widehat{h}\right|_{L_{x}}$ for all $x \in U$. By using partitions of unity it is easy to endow $L$ with a hermitian metric.

We say that a linear space $L$ is Nakano semi positive (resp., Nakano semi negative) if one can endow $L$ with a hermitian metric $h$ such that $L$ restricted to $\Omega:=W_{L} \backslash X_{\mathrm{sg}}$ is negative (resp., positive) with respect to $\left.h\right|_{\Omega}$ in the sense of Nakano [11]. This means that each point $x_{*} \in \Omega$ admits an open neighborhood $U \subset \Omega$ and $z=\left(z_{1}, \ldots, z_{n}\right)$ local coordinates centered at $x_{*}$ and an isomorphism $\left.L\right|_{U} \longrightarrow U \times \mathbb{C}^{r}$ such that for the matrix $\left(h_{j k}\right)$ we have at $x_{*}$ :

1) $\left(h_{j k}\left(x_{*}\right)\right)=\left(\delta_{j k}\right)=$ identity matrix,

2) $\left(d h_{j k}\left(x_{*}\right)\right)=0$, and

3) the hermitian form $\sum \frac{\partial^{2} h_{j k}}{\partial z_{\nu} \bar{\partial} z_{\mu}}\left(x_{*}\right) \xi_{j \nu} \bar{\xi}_{k \mu}$ is negative semi-definite (resp., positive semi-definite), for every $\xi \in T_{x^{*}} \Omega \otimes \mathbb{C}^{r}$.

Lemma 1. Every linear space over a Stein space is Nakano semi-positive and Stein.

Proof: First, let us recall some computational facts. Let $\Omega$ be a nonempty open subset of $\mathbb{C}^{n}$ and $E=\Omega \times \mathbb{C}^{r}$ a trivial holomorphic vector bundle of rank $r$ on $\Omega$. Consider a smooth function $h$ defined on $\Omega$ with values in the set of positively definite hermitian matrices of rank $r$. It defines a hermitian metric on $E$ by setting

$$
(x, \xi) \longmapsto{ }^{t} \xi h(x) \bar{\xi}
$$

and its $(1,1)$ curvature form has the expression

$$
i \Theta(E, h)=i \bar{\partial}\left(\bar{h}^{-1} \partial \bar{h}\right) .
$$


Besides, if $u$ is a real-valued smooth function on $\Omega$, then

$$
i \Theta\left(E, h e^{-u}\right)=i \Theta(E, h)+i \partial \bar{\partial} u \mathrm{I}_{r} .
$$

For the sake of clarity, let us mention the following correspondence between real $(1,1)$-forms and hermitian forms on an open subset of a complex euclidean space with coordinates $\left(w_{1}, \ldots, w_{q}\right)$. To a hermitian form

$$
g=\sum g_{j k} d w_{j} \otimes d \bar{w}_{k}
$$

it corresponds the $(1,1)$-form

$$
\omega:=-\frac{i}{2} \sum g_{j k} d w_{j} \wedge d \bar{w}_{k}
$$

so that $\omega(\alpha, \beta)=\operatorname{Im} g(\alpha, \beta)$, and, in particular $\omega(\alpha, \alpha)=g(\alpha, \alpha)$, which relates the complex hessian (or hermitian Levi form) and $(1,1)$-forms.

Now, it is straightforward to construct a hermitian metric $h$ on $L$ by using a partition of unity subordinated to a Stein open covering $\left\{U_{j}\right\}$ of $X$ by local embeddings of $\left.L\right|_{U_{j}} \subset U_{j} \times \mathbb{C}^{n_{j}}$. If $\varphi>0$ is a smooth, strictly plurisubharmonic, and exhaustion function on $X$, there is a rapidly increasing convex function $\chi:(0, \infty) \longrightarrow(0, \infty)$ such that the modified hermitian metric $h e^{-\chi(\varphi)}$ is as desired. Details of the proof are left to the interested reader.

The Stein property of the linear spaces is treated in [14] in a more general setting, namely the following result is proved.

Proposition 2. A linear space over a q-complete base space is q-complete. In particular, if $X$ is Stein, then $E$ is Stein, too.

The normalisation is such that "1-complete" means Stein. (The setting of vector bundles over $q$-complete spaces is treated in [17].)

The canonical line bundle $K_{X}$ on a complex space $X$ is as in [8]. Let $\pi: \hat{X} \longrightarrow X$ be a resolution of singularities of $X$ and put $K_{X}$ be the direct image through $\pi$ of the canonical sheaf on $\hat{X}$. From Lemma 1 we get the following result.

Corollary 6. Let $X$ be a Stein space. Then the linear space associated to $K_{X}$ or its dual $K_{X}^{\star}$ is Nakano semi-positive.

\section{Proof of Proposition 1}

First we recall a well-known criterion for Kähler completeness. 
Lemma 2. Let $(M, \omega)$ be a Kähler manifold and $\theta$ a smooth real-valued function on $M$ such that

$$
\omega \geq 2 i \cdot \partial \theta \wedge \bar{\partial} \theta
$$

Then, if $\delta$ represents the geodesic distance with respect to $\omega$, for arbitrary points $z_{1}, z_{2} \in M$, one has $\delta\left(z_{1}, z_{2}\right) \geq\left|\theta\left(z_{1}\right)-\theta\left(z_{2}\right)\right|$. In particular, if $\theta$ is exhaustive, then $\omega$ is complete.

Proposition 3. Let $X$ be a Stein space of pure dimension $n$ and let $D$ be an open subset of $X$ that is locally Stein at every boundary point of $\partial D \backslash X_{\mathrm{sg}}$.

Then there exists a smooth function $\phi: D \longrightarrow \mathbb{R}$ that is strictly plurisubharmonic and for every closed subset $T$ of $D$ whose closure in $X$ is disjoint from $X_{\mathrm{sg}}$, the restriction of $\phi$ to $T$ is exhaustive.

Proof: First by [1] we note that if $V \Subset X$ is a Stein open set, then there are finitely many discrete holomorphic mappings $\tau_{j}: V \longrightarrow \mathbb{C}^{n}$ and holomophic functions $f_{j}$ on $V, j=1, \ldots, m$, with the following properties.

The induced mappings $\tau_{j}: V \backslash\left\{f_{j}=0\right\} \longrightarrow \mathbb{C}^{n}$ are locally biholomorphic, therefore $\left(V \backslash\left\{f_{j}=0\right\}, \tau_{j}\right)$ becomes a domain over $\mathbb{C}^{n}$, and the intersection of all $\left\{f_{j}=0\right\}$ equals $V \cap X_{\mathrm{sg}}$.

Set $U=V \cap \Omega$ and $U_{j}=\left(V \backslash\left\{f_{j}=0\right\}\right) \cap \Omega$. Then $U_{j}$ is a Stein domain over $\mathbb{C}^{n}$ via $\left.\tau_{j}\right|_{U_{j}}$ because $V \backslash\left\{f_{j}=0\right\}$ is a Stein manifold and $U_{j}$ is locally Stein in $V \backslash\left\{f_{j}=0\right\}$. Let $\delta_{j}$ be the boundary distance of the domain $U_{j}$ over $\mathbb{C}^{n}$. Thus $-\log \delta_{j}$ is continuous and plurisubharmonic.

For $k \in \mathbb{N}=\{1,2, \ldots\}$ set $\theta_{j}^{(k)}=\max \left\{0,-\log \delta_{j}+k \log \left|f_{j}\right|\right\}$ that is defined on $U$ with values in $[0, \infty)$ and set

$$
\psi_{V}^{(k)}=\max \left\{\theta_{1}^{(k)}, \ldots, \theta_{m}^{(k)}\right\}: U \longrightarrow[0, \infty) .
$$

There is $N_{V} \in \mathbb{N}$ such that for every $k \in \mathbb{N}, k \geq N_{V}, \theta_{j}^{(k)}$ are continuous and plurisubharmonic on $U$ (see [16, Corollary 4.9]). In particular $\psi_{V}^{(k)}$ is continuous and plurisubharmonic on $U$. Observe also that $\psi_{V}^{(k)}(x)$ tends to infinity as $x \in U \backslash X_{\mathrm{sg}}$ goes to a point of $V \cap\left(\partial \Omega \backslash X_{\mathrm{sg}}\right)$.

One important feature of this construction is that, if we start with another Stein open set $W \Subset X$ to get $\psi_{W}^{(l)}$, then for every compact set $M \subset V \cap W$, there is $N_{V W} \in \mathbb{N}$ such that, for every $k, l \in \mathbb{N}$, $k, l \geq N_{V W}$, the difference function $\psi_{V}^{(k)}-\psi_{W}^{(l)}$ is bounded on $M \cap \Omega$. This follows by using Lemmata 4.5 and 4.8 , and Corollary 4.9 from [16].

Now choose a locally finite family $\left\{V_{\alpha}\right\}_{\alpha}$ of relatively compact Stein open subsets of $X$ that covers $\bar{\Omega}$ and either $V_{\alpha} \cap \partial \Omega \neq \emptyset$ or else $V_{\alpha} \Subset \Omega$. Then select open sets $V_{\alpha}^{\prime \prime} \Subset V_{\alpha}^{\prime} \Subset V_{\alpha}$ such that $\left\{V_{\alpha}^{\prime \prime}\right\}_{\alpha}$ still cover $\bar{\Omega}$. Fix 
functions $\mu_{\alpha} \in C^{0}(X, \mathbb{R})$ with compact support $K_{\alpha}, V_{\alpha}^{\prime} \subset K_{\alpha} \subset V_{\alpha}$, $\mu_{\alpha}=1$ on $V_{\alpha}^{\prime \prime}$ and $\mu_{\alpha}=-1$ on $\partial V_{\alpha}^{\prime}$. There are $k_{\alpha} \in \mathbb{N}$ such that setting $\psi_{\alpha}=\psi_{V_{\alpha}}^{\left(k_{\alpha}\right)}$ each difference function $\psi_{\alpha}-\psi_{\beta}$ is bounded on $K_{\alpha} \cap K_{\beta} \cap \Omega$.

Thus, if $\psi: X \longrightarrow[0, \infty)$ is strictly plurisubharmonic, continuous and exhaustive, there are constants $C_{\alpha} \geq 0$ (we take $C_{\alpha}=0$ if $V_{\alpha} \Subset \Omega$ ) and a strictly increasing convex function $\chi:[0, \infty) \longrightarrow[0, \infty)$ such that we can patch the continuous plurisubharmonic functions $\psi_{\alpha}+C_{\alpha} \mu_{\alpha}+$ $\chi\left(\left.\psi\right|_{V_{\alpha}}\right)$ defined on $V_{\alpha}$ to a continuous strictly plurisubharmonic function $\phi: \Omega \longrightarrow[0, \infty)$ by setting for $x \in \Omega$ :

$$
\varphi(x)=\chi(\psi(x))+\sup _{\alpha}\left\{\psi_{\alpha}(x)+C_{\alpha} \mu_{\alpha}(x)\right\} .
$$

This has the desired properties except smoothness. But this can be achieved since by [13, Satz 4.2] we can approximate $\phi$ in the $C^{0}$-topology by a smooth strictly plurisubharmonic function, whence the proof.

End of proof of Proposition 1: Without any loss in generality we may assume that $D$ is connected. Now, since every closed analytic subset of a Stein space of finite dimension is the common zero set of finitely many holomorphic functions (as it follows by a simple Baire argument and mathematical induction), there are $f_{1}, \ldots, f_{m}$ holomorphic functions on $X$ such that $X_{\mathrm{sg}}=\left\{x \in X ; f_{1}(x)=\cdots=f_{m}(x)=0\right\}$. Let $\alpha:=$ $\left|f_{1}\right|^{2}+\cdots+\left|f_{m}(x)\right|^{2}$. Let $U$ and $V$ be open neighborhoods of $X_{\mathrm{sg}}$ in $X$ with $\bar{V} \subset U$ and such that $\alpha \leq 1$ on $U$. Then select $\rho \in C^{\infty}(X, \mathbb{R})$ such that $\rho \geq 0$ on $X, \rho=1$ on $V$, and $\operatorname{Supp}(\rho) \subset U$. Define $\sigma=(1-\rho+\rho \alpha) / 3$ so that $0 \leq \sigma<1 / e$ on $X, \sigma=\left(\left|f_{1}\right|^{2}+\cdots+\left|f_{m}(x)\right|^{2}\right) / 3$ on $V$, and $\sigma$ is constant and equals $1 / 3$ on $X \backslash U$. Therefore $\sigma$ is plurisubharmonic on $V \cup(X \backslash \operatorname{Supp}(\rho))$.

Put $\theta=\log \sigma$. Thus $\theta<-1$ on $X$, and if $\left\{x_{\nu}\right\}$ is a sequence of points in $D \backslash X_{\mathrm{sg}}$ converging to a point $x_{\star} \in D \cap X_{\mathrm{sg}}$, then $\left\{-\theta\left(x_{\nu}\right)\right\}$ tends to infinity.

Let $\gamma>0$ be a smooth strictly plurisubharmonic exhaustion function on $X$. We may assume that

$$
i \partial \bar{\partial} \gamma+\frac{1}{2 \sqrt{-\theta}} i \partial \bar{\partial} \theta
$$

is semi-positive (replace $\gamma$ by $\chi(\gamma)$ for a suitable smooth strictly increasing convex function $\chi$ from $(0, \infty)$ into $(0, \infty)$, if necessary).

Now we claim that the following closed $(1,1)$-form $\omega$ defined on $D \backslash X_{\mathrm{sg}}$ is Kähler complete, where

$$
\omega=i \partial \bar{\partial}\left(\phi^{2}+\gamma^{2}+\gamma-\sqrt{-\theta}\right) .
$$


Indeed, first it is easily checked that

$$
i \partial \bar{\partial}(-\sqrt{-\theta})=\frac{1}{2 \sqrt{-\theta}} i \partial \bar{\partial} \theta+\frac{1}{4}(-\theta)^{-3 / 2} i \partial \theta \wedge \bar{\partial} \theta,
$$

so that we deduce immediately that $\omega$ is positively definite.

To check the completeness of $\omega$ notice that, if $\delta$ denotes the geodesic distance induced by $\omega$, for arbitrary points $z_{1}, z_{2} \in D \backslash X_{\text {sg }}$ the following inequality holds:

$\delta\left(z_{1}, z_{2}\right) \geq \max \left\{\left|\phi\left(z_{1}\right)-\phi\left(z_{2}\right)\right|,\left|\gamma\left(z_{1}\right)-\gamma\left(z_{2}\right)\right|, \sqrt{2} \mid\left(-\theta\left(z_{1}\right)\right)^{\frac{1}{4}}-\left(-\theta\left(z_{2}\right)^{\frac{1}{4}} \mid\right\}\right.$.

We then conclude since for a sequence $\left\{x_{j}\right\}_{j}$ of points in $D \backslash X_{\text {sg }}$ converging to a point of $\partial D \cup X_{\mathrm{sg}}$, at least one of the sequences $\left\{\phi\left(x_{j}\right)\right\}_{j}$, $\left\{\gamma\left(x_{j}\right)\right\}_{j}$, or $\left\{-\theta\left(x_{j}\right)\right\}_{j}$ is unbounded.

\section{Proof of Theorem 1}

First we recall Demailly's version of the $\bar{\partial}$ problem with singular weights on Kähler complete manifolds [3].

Theorem 2. Let $(E, h)$ be a semi-positive hermitian holomorphic line bundle over a complete Kähler manifold $(M, \omega)$ of dimension $n$, that is $i \Theta(E, h) \geq 0$. Consider a function $\varphi: M \longrightarrow \mathbb{R} \cup\{-\infty\}$ that is $C^{\infty}$ outside a discrete set $S$ of $M$ and near every point $p \in S$,

$$
\varphi(z)=A_{p} \log |z|^{2},
$$

where $A_{p}$ is a positive constant and $z=\left(z_{1}, \ldots, z_{n}\right)$ are local coordinates centered at $p$. Assume that $i \Theta\left(E, h e^{-\varphi}\right)=i \Theta(E, h)+i \partial \bar{\partial} \varphi \geq 0$ on $M \backslash S$ and let $\lambda: M \longrightarrow[0,1]$ be a continuous function such that on $M \backslash S$ one has

$$
i \Theta(E, h)+i \partial \bar{\partial} \varphi \geq \lambda \omega .
$$

Then, for each $C^{\infty}$ form $v$ of type $(n, 1)$ with values in $E$ on $M$ such that $\bar{\partial} v=0$ and

$$
\int_{M} \frac{1}{\lambda}|v|^{2} e^{-\varphi} d V_{\omega}<\infty
$$

there is a $C^{\infty}$ form $u$ of type $(n, 0)$ with values in $E$ on $M$ such that $\bar{\partial} u=v$ and

$$
\int_{M}|u|^{2} e^{-\varphi} d V_{\omega} \leq \int_{M} \frac{1}{\lambda}|v|^{2} e^{-\varphi} d V_{\omega} .
$$

Now we start with the proof of Theorem 1 . Since on $D \backslash X_{\mathrm{sg}}$ we have the natural isomorphism of holomorphic vector bundles $E \simeq\left(E \otimes K_{X}^{*}\right) \otimes$ $K_{X}$ the idea is to use Theorem 2 to solve a $\bar{\partial}$ problem for $(0,1)$ forms with coefficients in $F:=E \otimes K_{X}^{\star}$ on $D \backslash X_{\text {sg }}$ and due to Nakano semi positivity of that bundle (see Lemma 1), to check the hypotheses of 
the theorem reduces to a proper choice of a smooth convex increasing function in order to satisfy the curvature inequalities and convergence of a certain integral. Since $D$ is normal, the holomorphic section we construct on $D \backslash X_{\mathrm{sg}}$ with values in $E$ extends to a holomorphic section in $E$ over $D$.

To proceed, let $\left\{x_{j}\right\}_{j}$ be a sequence of points of $D \backslash X_{\mathrm{sg}}$ whose set of accumulation points, which is a closed subset of $X$, and by hypothesis is contained in $\partial D$ and disjoint from $X_{\mathrm{sg}}$. Then, select relatively compact open neighborhoods $V_{j}$ and $U_{j}$ of $x_{j}$ in $D \backslash X_{\mathrm{sg}}$ such that $\bar{U}_{j}$ are mutually disjoint and contained in $D \backslash X_{\mathrm{sg}}$ and $\bar{V}_{j} \subset U_{j}$. We choose $U_{j}$ such that $T:=\cup_{j} \bar{U}_{j}$ is a closed subset of $D$, and whose closure in $X$ is disjoint from $X_{\mathrm{sg}}$. Moreover, we may assume that, for each index $j$, $U_{j}$ is the domain of a coordinate chart centered at $x_{j}$ and $z^{(j)}$ be complex coordinates centered at $x_{j}$ on $U_{j}$.

Now, let $\rho_{j}$ be a smooth function whose support lies in $U_{j}$ and equals 1 on $V_{j}$. We define a smooth section $\sigma$ in $E$ over $D \backslash X_{\text {sg }}$ by $\sigma=\sum \rho_{j} \xi_{j}$. This induces a holomorphic form $\tilde{\sigma}$ of type $(n, 0)$ on $D \backslash X_{\mathrm{sg}}$ with coefficients in $F$, where $n$ is the complex dimension of $X$. Consider the smooth $(n, 1)$-form with coefficients in $F$ defined by $v=\bar{\partial} \sigma$. Then $v$ has support in $T^{\prime}:=\cup_{j}\left(\bar{U}_{j} \backslash V_{j}\right) \subset T$. Now solve the $\bar{\partial}$ equation $\bar{\partial} u=v$ with singular estimates and for that we choose in the statement of Theorem 2 the constants $A_{p}=n$ and obtain a smooth $(n, 0)$ form $u$ with coefficients in $F$ such that $u\left(x_{j}\right)=0$ for all indices $j$. Then $\tilde{\tau}=\tilde{\sigma}-u$ induces a holomorphic section $\tau$ in $E$ over $D \backslash X_{\text {sg }}$ with $\sigma\left(x_{j}\right)=\xi_{j}$ for all indices $j$. Since $D$ is normal, the extension of $\tau$ to $D$ is as desired.

Now, we go on as follows. Keeping the notations from $\S 3$ (proof of Proposition 1), the function $\gamma$ is positive, smooth of class $C^{\infty}$, strictly plurisubharmonic and exhaustive for $X$, and we set:

$$
\Phi_{1}=\phi^{2}+\left.\gamma^{2}\right|_{D \backslash X_{\mathrm{sg}}} \text { and } \Phi_{2}=\left.(\gamma-\sqrt{-\theta})\right|_{D \backslash X_{\mathrm{sg}}} .
$$

Let $\chi:[0, \infty) \longrightarrow(0, \infty)$ be an increasing, smooth of class $C^{\infty}$, and convex function, to be chosen later in proof. We put

$$
\omega_{\chi}=\omega+i \partial \bar{\partial} \chi\left(\Phi_{1}\right) \text {, where } \omega=i \partial \bar{\partial}\left(\Phi_{1}+\Phi_{2}\right) .
$$

Consider the singular weight function

$$
\Phi_{0}=\sum n \rho_{j} \cdot \log \left\|z^{(j)}\right\|^{2},
$$

where $\rho_{j}=1$ on $V_{j}, \rho_{j} \geq 0$ on $X$, and its support lies in $U_{j}$, where $U_{j}$ and $V_{j}$ are as above. Define

$$
\Psi=\Phi_{0}+\Phi_{2}+3 \chi\left(\Phi_{1}\right)
$$

and let the singular hermitian metric on $F$ be $h e^{-\Psi}$. 
We would like to apply Theorem 2 for $\lambda$ the constant function, $\lambda=1$, and for that we need to select $\chi$ carefully; we wish the inequality

$$
i \partial \bar{\partial} \Psi \geq \omega_{\chi},
$$

and, in order to achieve this we require that $i \partial \bar{\partial} \chi\left(\Phi_{1}\right) \geq i \partial \bar{\partial} \Phi_{1}$, a condition that is fulfilled if we impose that $\chi-$ id be positive, increasing, and convex, and the inequality

$$
i \partial \bar{\partial} \Phi_{0}+i \partial \bar{\partial} \chi\left(\Phi_{1}\right) \geq 0
$$

that can be easily realized if $\chi^{\prime}$ is large enough taking into account the computations from $\S 3$.

Then we need the convergence of the integral

$$
\int_{D \backslash X_{\mathrm{sg}}}|v|^{2} e^{-\Psi} d V_{\chi}
$$

which, as a matter of fact, is an integral over $T^{\prime}$ and $\Phi_{0}$ and $\Phi_{2}$ are smooth there, and where $d V_{\chi}$ is the volume element associated to the complete Kähler form $\omega_{\chi}$. By straightforward computations we obtain

$$
d V_{\chi} \leq C \cdot\left(1+\chi^{\prime} \circ \Phi_{1}+\chi^{\prime \prime} \circ \Phi_{1}\right)^{n} d V
$$

for some positive continuous function $C$ on $D \backslash X_{\mathrm{sg}}$, where $d V$ is the volume element associated to the Kähler form $\omega$. Therefore, the convergence will follow by using the following lemma due to Demailly $[\mathbf{4}$, p. 374].

Lemma 3. For any continuous function $\lambda:[0, \infty) \longrightarrow(0, \infty)$, there exists a $C^{\infty}$-smooth convex function $\chi:[0, \infty) \longrightarrow \mathbb{R}$ such that

$$
\min \left(\chi, \chi^{\prime}, \chi^{\prime \prime}\right) \geq \lambda \text { and }\left(1+\chi^{\prime}+\chi^{\prime \prime}\right)^{n} \epsilon^{-\chi} \leq 1 / \lambda .
$$

The proof of Theorem 1 is completed.

\section{References}

[1] A. Andreotti and R. Narasimhan, Oka's Heftungslemma and the Levi problem for complex spaces, Trans. Amer. Math. Soc. 111(2) (1964), 345-366. DOI: $10.2307 / 1994247$.

[2] M. Coltoiu And K. Diederich, On Levi's problem on complex spaces and envelopes of holomorphy, Math. Ann. 316(1) (2000), 185-199. DOI: 10.1007/ s002080050009.

[3] J.-P. Demailly, Estimations $L^{2}$ pour l'opérateur $\bar{\partial}$ d'un fibré vectoriel holomorphe semi-positif au-dessus d'une variété kählérienne complète, Ann. Sci. École Norm. Sup. (4) 15(3) (1982), 457-511.

[4] J.-P. Demailly, "Complex Analytic and Differential Geometry" (2012). Available at: https://www-fourier.ujf-grenoble.fr/ demailly/manuscripts/ag book.pdf. 
[5] G. Fischer, "Complex Analytic Geometry", Lecture Notes in Mathematics 538, Springer-Verlag, Berlin-New York, 1976. DOI : 10.1007/BFb0080338.

[6] J. E. Fornæss, and R. NARAsimhan, The Levi problem on complex spaces with singularities, Math. Ann. 248(1) (1980), 47-72. DOI : 10.1007/BF01349254.

[7] K. Fritzsche and H. Grauert, "From Holomorphic Functions to Complex Manifolds", Graduate Texts in Mathematics 213, Springer-Verlag, New York, 2002. DOI : $10.1007 / 978-1-4684-9273-6$.

[8] H. Grauert and O. Riemenschneider, Verschwindungssätze für analytische Kohomologiegruppen auf komplexen Räumen, Invent. Math. 11(4) (1970), 263-292. DOI : $10.1007 / \mathrm{BF} 01403182$.

[9] A. Hirschowitz, Un exemple concernant le prolongement analytique, $C . R$. Acad. Sci. Paris Sér. A-B 275 (1972), A1231-A1233.

[10] L. Hörmander, "An Introduction to Complex Analysis in Several Variables", Third edition, North-Holland Mathematical Library 7, North-Holland Publishing Co., Amsterdam, 1990.

[11] S. Nakano, On complex analytic vector bundles, J. Math. Soc. Japan 7(1) (1955), 1-12. DOI : $10.2969 / j m s j / 00710001$.

[12] O. Preda, Locally Stein open subsets in normal Stein spaces, J. Geom. Anal. 25(4) (2015), 2759-2766. DOI : 10.1007/s12220-014-9535-9.

[13] R. Richberg, Stetige streng pseudokonvexe Funktionen, Math. Ann. 175(4) (1968), 257-286. DOI : 10.1007/BF02063212.

[14] V. VÂJÂITU, Some convexity properties of morphisms of complex spaces, Math. Z. 217(1) (1994), 215-245. DOI : 10.1007/BF02571943.

[15] V. VÂJÂtTu, Compact sets with vanishing cohomology in Stein spaces and domains of holomorphy, Ann. Sc. Norm. Super. Pisa Cl. Sci. (5) 12(3) (2013), $665-685$.

[16] V. VÂJÂITU, A Levi problem for convexity with corners, Complex Var. Elliptic Equ. 61(8) (2016), 1187-1197. DOI: 10.1080/17476933.2016.1153634.

[17] V. Villani, Fibrati vettoriali olomorfi su una varietà complessa $q$-completa, Ann. Scuola Norm. Sup. Pisa (3) 20 (1966), 15-23.

Université des Sciences et Technologies de Lille 1, Laboratoire Paul Painlevé, Bât. M2, F-59655 Villeneuve d'Ascq Cedex, France

E-mail address: viorel.vajaitu@univ-lille.fr

Primera versió rebuda el 27 de novembre de 2017, darrera versió rebuda el 3 de setembre de 2018. 